Research Article

\title{
Mixture of Lindley and Lognormal Distributions: Properties, Estimation, and Application
}

\author{
A. S. Al-Moisheer \\ Department of Mathematics, College of Science, Jouf University, P.O. Box 848, Sakaka 72351, Saudi Arabia \\ Correspondence should be addressed to A. S. Al-Moisheer; asalmoisheer@ju.edu.sa
}

Received 26 June 2021; Revised 23 July 2021; Accepted 29 September 2021; Published 29 December 2021

Academic Editor: Badr Saad. T. Alkaltani

Copyright (c) 2021 A. S. Al-Moisheer. This is an open access article distributed under the Creative Commons Attribution License, which permits unrestricted use, distribution, and reproduction in any medium, provided the original work is properly cited.

\begin{abstract}
Finite mixture models provide a flexible tool for handling heterogeneous data. This paper introduces a new mixture model which is the mixture of Lindley and lognormal distributions (MLLND). First, the model is formulated, and some of its statistical properties are studied. Next, maximum likelihood estimation of the parameters of the model is considered, and the performance of the estimators of the parameters of the proposed models is evaluated via simulation. Also, the flexibility of the proposed mixture distribution is demonstrated by showing its superiority to fit a well-known real data set of 128 bladder cancer patients compared to several mixture and nonmixture distributions. The Kolmogorov Smirnov test and some information criteria are used to compare the fitted models to the real dataset. Finally, the results are verified using several graphical methods.
\end{abstract}

\section{Introduction}

In most reliability applications, data is modeled by a single parametric distribution. However, in many situations, a population can be divided into a number of subpopulations each representing a different type of failure. Finite mixture models play an important role in modeling such heterogeneous data. Applications of mixture models are especially in clustering and classification, see, for example, Everitt and Hand [1], McLachlan and Peel [2], McLachlan and Basford [3], Titterington et al. [4], Lindsay [5], McLachlan and Krishnan [6], Al-Moisheer et al. [7, 8], and Al-Moisheer [9, 10]. In this paper, we will introduce a finite mixture of Lindley and lognormal distributions (MLLND). The motivation of suggesting this mixture comes from the importance of its component distributions. The one-parameter Lindley distribution was introduced by Lindley [11, 12], and then Ghitany et al. [13] illustrated its importance in lifetesting and reliability applications. With regards to one component lognormal distribution, it has found important applications in a wide variety of fields; (see, Kim and Yum [14] and Lin et al. [15]). In the literature, work has been done on mixture models having the Lindley distribution as one of its components, see, for example, Al-Moisheer et al. (Al-Moisheer et al. [16], Al-Moisheer et al. [17]) for the mixture of two one-parameter Lindley distribution and the mixture of Lindley and inverse Weibull distributions, respectively. Also, Daghestani et al. [18] considered the mixture of Lindley and Weibull distributions. In this paper, we will introduce a new mixture distribution, namely, the finite mixture of Lindley and lognormal distribution (MLLND). This paper is organized as follows. In Section 2, we obtain the new model and derive some of its properties. In Sections 3 and 4 , we derive the probability density function of the order statistics and the equations required to obtain the maximum likelihood estimation of the model parameters. In Section 5 , the flexibility of the proposed model is illustrated by showing its ability to provide the best fit for a well-known real data set compared to six competitive models. Finally, In Section 6, we draw a conclusion. 


\section{Model Formulation and Some Properties}

The MLLND has the following probability density function (pdf)

$$
\begin{aligned}
f(x ; \Theta) & =p_{1} f_{1}\left(x ; \Theta_{1}\right)+p_{2} f_{2}\left(x ; \Theta_{2}\right), 0<p_{1}, p_{2}<1, p_{1}+p_{2} \\
& =1, x \geq 0
\end{aligned}
$$

whereas the pdf of the Lindley component is given by

$$
f_{1}\left(x ; \Theta_{1}\right)=\frac{\theta^{2}}{\theta+1}(1+x) e^{-\theta x}, x \geq 0, \theta>0 .
$$

The pdf of the lognormal component is given by

$$
f_{2}\left(x ; \Theta_{2}\right)=\frac{1}{\sqrt{2 \pi} \sigma x} e^{-\frac{1}{2}\left(\frac{\log x-\mu}{\sigma}\right)^{2}}, x \geq 0,-\infty<\mu<\infty, \sigma>0,
$$

$\Theta=\left(p_{1}, \theta, \mu, \sigma\right), \Theta_{1}=(\theta)$, and $\Theta_{2}=(\mu, \sigma)$.

Evidently, the cumulative distribution function (cdf) of the MLLND is given by

$$
F(x ; \Theta)=p_{1} F_{1}\left(x ; \Theta_{1}\right)+p_{2} F_{2}\left(x ; \Theta_{2}\right)
$$

where

$$
\begin{aligned}
& F_{1}\left(x ; \Theta_{1}\right)=1-\frac{\theta+1+\theta x}{\theta+1} e^{-\theta x}, x \geq 0, \theta>0, \\
& F_{2}\left(x ; \Theta_{2}\right)=\Phi\left(\frac{\log x-\mu}{\sigma}\right), x \geq 0,-\infty<\mu<\infty, \sigma>0,
\end{aligned}
$$

with $\Phi($.$) referring to the cdf of the standard normal$ distribution.

Ghitany et al. [13] and Shanker et al. [19] displayed some properties of the LD in (2), while properties of the LND in (3) were given, for example, by Crow and Shimizu [20] and Johnson et al. [21]. In this section, we introduce some properties of the MLLND by mixing the results of the LD and LND.

2.1. Mean and Variance. The mean of the MLLND in (1) is simply given by

$$
\begin{aligned}
E(X)= & p_{1}\left[\frac{\theta+2}{\theta(\theta+1)}\right]+p_{2} e^{\mu+\frac{\sigma^{2}}{2}}, \text { for } x \geq 0, \theta, \sigma>0, \\
& -\infty<\mu<\infty,
\end{aligned}
$$

while the variance is given by

$$
\begin{aligned}
\operatorname{Var}(X)= & \frac{p_{1}}{\theta^{2}(\theta+1)}\left[2(\theta+3)-p_{1}(\theta+2) \theta\right] \\
& +p_{2} e^{2 \mu+\sigma^{2}}\left[e^{\sigma^{2}}-p_{2}\right]-2 p_{1} p_{2}\left(\frac{\theta+2}{\theta(\theta+1)}\right) \mathrm{e}^{\mu}++^{\frac{\sigma 2}{2}}, \\
& \text { for } \mathrm{x} \geq 0, \theta, \sigma>0,-\infty<\mu<\infty .
\end{aligned}
$$

Also, the $r$ th moments of the MLLND is given by

$$
E\left(X^{r}\right)=\frac{p_{1}(\theta+r+1) \Gamma(r+1)}{\theta^{r}(\theta+1)}+p_{2} e^{r \mu+\frac{r^{2} \sigma^{2}}{2}}, \text { for } r=1,2,3, \cdots .
$$

2.2. Mode and Median. It can be shown that the equations for obtaining the modes and median of the MLLND, respectively, are

$$
p_{1} \frac{\theta^{2} e^{-\theta x}}{\theta+1}[1-\theta(1+x)]-p_{2} \frac{1}{\sqrt{2 \pi} \sigma^{3} x^{2}} e^{-\frac{1}{2}\left(\frac{\log x-\mu}{\sigma}\right)^{2}}\left[\sigma^{2}+(\log x-\mu)\right]=0,
$$

and

$$
p_{1}\left(1-\frac{(\theta+1+\theta x) e^{-\theta x}}{\theta+1}\right)+p_{2} \Phi\left(\frac{\log x-\mu}{\sigma}\right)=0.5
$$

Figure 1(a) shows the pdf of the MLLND unimodal case at the choice of parameters $\Theta=\left(p_{1}=0.5, \theta=0.95, \mu=0.25\right.$, $\sigma=0.25)$ with the values of mode and median (1.1623, 1.2712), respectively. Also, Figure 1(b) shows the shape of the pdf in the MLLND bimodal case at the choice of parameters $\Theta=\left(p_{1}=0.5, \theta=2.95, \mu=0.85, \sigma=0.25\right)$ with the values of mode and median $((1.4370,2.1430), 1.4542)$, respectively. For plotting the pdf of the LD and LND in $R$, we use the function dlindley() and dlnorm(), respectively. The package rootSolve() in $R$ is used for modes and median of the MLLND.

2.3. Reliability and Failure Rate Functions. The reliability function of the MLLND is given by

$$
R(x)=p_{1}\left[\frac{\theta+1+\theta x}{\theta+1} e^{-\theta x}\right]+p_{2}\left[1-\Phi\left(\frac{\log x-\mu}{\sigma}\right)\right], x \geq 0 .
$$

By using (3) and (4), the hazard rate function (HRF) of the MLLND is given by

$$
r(x)=\frac{p_{1}\left(\theta^{2} / \theta+1\right)(1+x) e^{-\theta x}+p_{2}(1 / \sqrt{2 \pi} \sigma x) e^{-1 / 2(\log x-\mu / \sigma)^{2}}}{p_{1}\left((\theta+1+\theta x / \theta+1) e^{-\theta x}\right)+p_{2}(1-\Phi(\log x-\mu / \sigma))}, x \geq 0,
$$

which can be written by using the result in AL-Hussaini and Sultan [22], as 


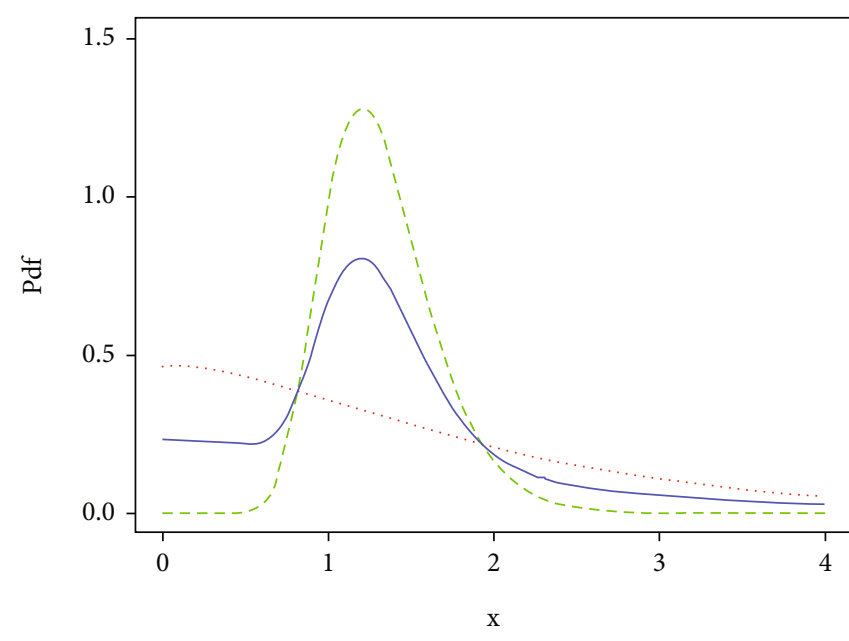

(a)

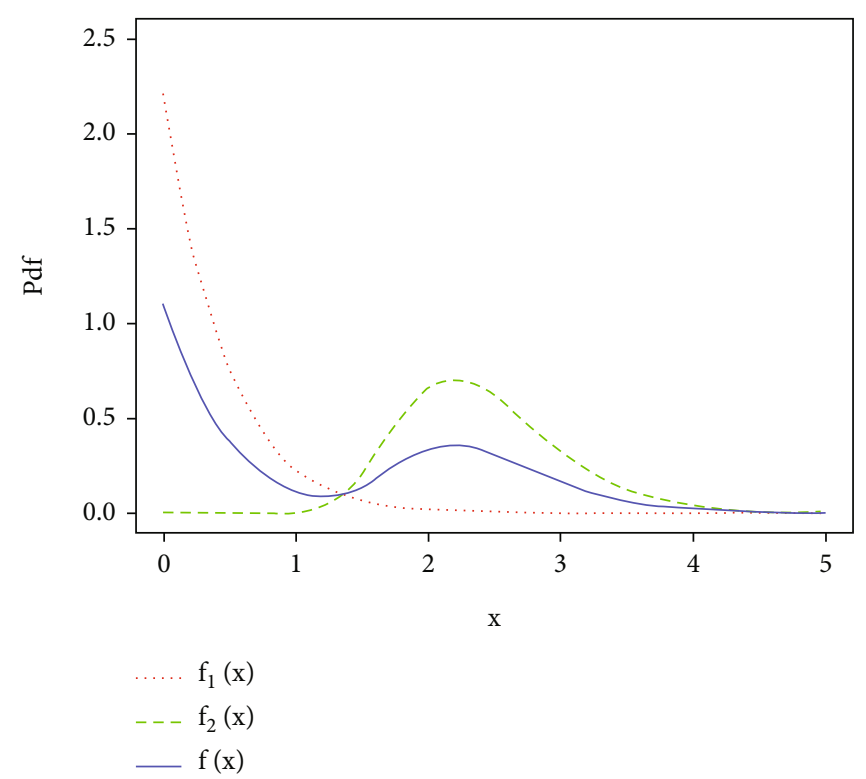

(b)

Figure 1: pdf plot of MLLND with (a) $\Theta=\left(p_{1}=0.5, \theta=0.95, \mu=0.25, \sigma=0.25\right)$ (b) $\Theta=\left(p_{1}=0.5, \theta=2.95, \mu=0.85, \sigma=0.25\right)$.

$$
r(x)=h(x) r_{1}(x)+(1-h(x)) r_{2}(x)
$$

where

$$
\begin{aligned}
h(x) & =\frac{1}{1+\left(p_{2} R_{2}(x) / p_{1} R_{1}(x)\right)}, r_{i}(x)=\frac{f_{i}(x)}{R_{i}(x)}, i=1,2, R_{1}(x) \\
& =\left(\frac{\theta+1+\theta x}{\theta+1} e^{-\theta x}\right) \text { and } R_{2}(x)=1-\Phi\left(\frac{\log x-\mu}{\sigma}\right) .
\end{aligned}
$$

The HRF of the MLLND in (12) achieves the following limits.

\section{Lemma 1.}

$$
\begin{aligned}
& \lim _{x \rightarrow 0} r(x)=\frac{p_{1} \theta^{2}}{\theta+1}, \\
& \lim _{x \longrightarrow \infty} r(x)=0 .
\end{aligned}
$$

Proof. To prove the first part of the limits, using the equation (13), we get

$$
\lim _{x \longrightarrow 0} h(x)=p_{1} \text {, and } \lim _{x \rightarrow 0}(1-h(x))=p_{2} .
$$

Then, we have

$$
\lim _{x \longrightarrow 0} r_{1}(x)=\frac{p_{1} \theta^{2}}{\theta+1}, \text { and } \lim _{x \longrightarrow 0} r_{2}(x)=0,
$$

and thus (15) is proved.
Also, to prove (16),

$$
\begin{gathered}
\lim _{x \longrightarrow \infty} r(x)=\lim _{x \longrightarrow \infty} \frac{f(x)}{1-F(x)}=-\lim _{x \longrightarrow \infty} \frac{f^{\prime}(x)}{f(x)},\left(f^{\prime}(x)=\frac{d f(x)}{d x}\right), \\
\text { where, } f^{\prime}(x)=p_{1} f^{\prime}{ }_{1}(x)+p_{2} f^{\prime}{ }_{2}(x) .
\end{gathered}
$$

It follows from (19) and (20) that

$$
\lim _{x \longrightarrow \infty} r(x)=-\lim _{x \longrightarrow \infty} \frac{p_{1} f_{1}^{\prime}(x)+p_{2} f_{2}^{\prime}(x)}{p_{1} f_{1}(x)+p_{2} f_{2}(x)} .
$$

It follows that

$$
\lim _{x \longrightarrow \infty} r(x)=0 .
$$

For more details, see Sultan [23], Sultan and Al-Moisheer [24], and Al-Moisheer et al. (Al-Moisheer et al. [16]).

2.4. Skewness, Kurtosis, and the Coefficient of Variation. The coefficient of skewness $(S k)$, the coefficient of kurtosis $(K u)$, and the coefficient of variation $(C v)$ of the MLLND distribution are given by, respectively,

$$
\begin{aligned}
S k= & \frac{E\left(X^{3}\right)-3 \mu \sigma^{2}-\mu^{3}}{\sigma^{3}},=\left(\frac{6 p_{1}(\theta+4)}{\theta^{3}(\theta+1)}\right)-\left(\frac{p_{1}(\theta+2)}{\theta(\theta+1)}+p_{2} e^{\mu+\frac{\sigma^{2}}{2}}\right)^{3} \\
& +p_{2} e^{3 \mu+\frac{\theta \sigma^{2}}{2}}-3\left(\frac{2 p_{1}(\theta+3)}{\theta^{2}(\theta+1)}-\left(\frac{p_{1}(\theta+2)}{\theta(\theta+1)}+p_{2} e^{\mu+\frac{\sigma^{2}}{2}}\right)^{2}+p_{2} e^{2\left(\mu+\sigma^{2}\right)}\right) \\
& \cdot\left(\frac{p_{1}(\theta+2)}{\theta(\theta+1)}+p_{2} e^{\mu+\frac{\sigma^{2}}{2}}\right) /\left(\frac{2 p_{1}(\theta+3)}{\theta^{2}(\theta+1)}-\left(\frac{p_{1}(\theta+2)}{\theta(\theta+1)}+p_{2} e^{\mu+\frac{\sigma^{2}}{2}}\right)^{2}+p_{2} e^{2\left(\mu+\sigma^{2}\right)}\right)^{\frac{3}{2}},
\end{aligned}
$$


TABLE 1: Results of the mean, standard deviation, coefficient of variation, skewness, and kurtosis for the MLLND at the choice $\left(p_{1}=0.5 ; \theta=0.1 ; \sigma=3\right)$.

\begin{tabular}{lccccc}
\hline$\mu$ & Mean & Stand.Dev. & Cv & Sk & Ku \\
\hline 1 & 124.013 & 15574.6 & 125.589 & $1.03165 \times 10^{6}$ & $8.62356 \times 10^{15}$ \\
2 & 334.237 & 42336.1 & 126.665 & $1.03165 \times 10^{6}$ & $8.62354 \times 10^{15}$ \\
3 & 905.688 & 115081 & 127.065 & $1.03165 \times 10^{6}$ & $8.62353 \times 10^{15}$ \\
4 & 2459.05 & 312824 & 127.213 & $1.03165 \times 10^{6}$ & $8.62353 \times 10^{15}$ \\
5 & 6681.53 & 850343 & 127.268 & $1.03165 \times 10^{6}$ & $8.62353 \times 10^{15}$ \\
6 & 18159.4 & $2.31147 \times 10^{6}$ & 127.288 & $1.03165 \times 10^{6}$ & $8.62353 \times 10^{15}$ \\
7 & 49359.6 & $6.28324 \times 10^{6}$ & 127.295 & $1.03165 \times 10^{6}$ & $8.62353 \times 10^{15}$ \\
8 & 134170 & $1.70796 \times 10^{7}$ & 127.298 & $1.03165 \times 10^{6}$ & $8.62353 \times 10^{15}$ \\
9 & 364710 & $4.64272 \times 10^{7}$ & 127.299 & $1.03165 \times 10^{6}$ & $8.62353 \times 10^{15}$ \\
10 & 991381 & $1.26202 \times 10^{8}$ & 127.299 & $1.03165 \times 10^{6}$ & $8.62353 \times 10^{15}$ \\
\hline
\end{tabular}

TABLE 2: Results of the mean, standard deviation, coefficient of variation, skewness, and kurtosis for the MLLND at the choice $\left(p_{1}=0.5 ; \theta=0.5 ; \sigma=3\right)$.

\begin{tabular}{lccccc}
\hline$\mu$ & Mean & Stand.Dev. & Cv & Sk & Ku \\
\hline 1 & 131.891 & 15574.5 & 118.086 & $1.03166 \times 10^{6}$ & $8.62368 \times 10^{15}$ \\
2 & 342.116 & 42336.0 & 123.747 & $1.03165 \times 10^{6}$ & $8.62359 \times 10^{15}$ \\
3 & 913.567 & 115081 & 125.969 & $1.03165 \times 10^{6}$ & $8.62355 \times 10^{15}$ \\
4 & 2466.93 & 312824 & 126.807 & $1.03165 \times 10^{6}$ & $8.62354 \times 10^{15}$ \\
5 & 6689.41 & 850343 & 127.118 & $1.03165 \times 10^{6}$ & $8.62353 \times 10^{15}$ \\
6 & 18167.3 & $2.31147 \times 10^{6}$ & 127.233 & $1.03165 \times 10^{6}$ & $8.62353 \times 10^{15}$ \\
7 & 49367.4 & $6.28324 \times 10^{6}$ & 127.275 & $1.03165 \times 10^{6}$ & $8.62353 \times 10^{15}$ \\
8 & 134178 & $1.70796 \times 10^{7}$ & 127.290 & $1.03165 \times 10^{6}$ & $8.62353 \times 10^{15}$ \\
9 & 364718 & $4.64272 \times 10^{7}$ & 127.296 & $1.03165 \times 10^{6}$ & $8.62353 \times 10^{15}$ \\
10 & 991389 & $1.26202 \times 10^{8}$ & 127.298 & $1.03165 \times 10^{6}$ & $8.62353 \times 10^{15}$ \\
\hline
\end{tabular}

$$
\begin{aligned}
K u= & \frac{E\left(X^{4}\right)-4 \mu E\left(X^{3}\right)+6 \mu^{2} \sigma^{2}+3 \mu^{4}}{\sigma^{4}},=\frac{24 p_{1}(\theta+5)}{\theta^{4}(\theta+1)} \\
& -4\left(\frac{6 p_{1}(\theta+4)}{\theta^{3}(\theta+1)}+p_{2} e^{3 \mu+\frac{9 \sigma^{2}}{2}}\right)\left(\frac{p_{1}(\theta+2)}{\theta(\theta+1)}+p_{2} e^{\mu+\frac{\sigma^{2}}{2}}\right) \\
& +3\left(\frac{p_{1}(\theta+2)}{\theta(\theta+1)}+p_{2} e^{\mu+\frac{\sigma^{2}}{2}}\right)^{4}+p_{2} e^{4 \mu+8 \sigma^{2}} \\
& +6\left(\frac{p_{1}(\theta+2)}{\theta(\theta+1)}+p_{2} e^{\mu+\frac{\sigma^{2}}{2}}\right)^{2}\left(\frac{2 p_{1}(\theta+3)}{\theta^{2}(\theta+1)}\right. \\
& \left.-\left(\frac{p_{1}(\theta+2)}{\theta(\theta+1)}+p_{2} e^{\mu+\frac{\sigma^{2}}{2}}\right)^{2}+p_{2} e^{2\left(\mu+\sigma^{2}\right)}\right)^{/}\left(\frac{2 p_{1}(\theta+3)}{\theta^{2}(\theta+1)}\right. \\
& \left.-\left(\frac{p_{1}(\theta+2)}{\theta(\theta+1)}+p_{2} e^{\mu+\frac{\sigma^{2}}{2}}\right)^{2}+p_{2} e^{2\left(\mu+\sigma^{2}\right)}\right)^{2},
\end{aligned}
$$

$C v=\frac{\sigma}{\mu}=\frac{\sqrt{\left(2 p_{1}(\theta+3) / \theta^{2}(\theta+1)-\left(\left(p_{1}(\theta+2) / \theta(\theta+1)\right)+p_{2} e^{\mu+\left(\sigma^{2} / 2\right)}\right)^{2}+p_{2} e^{2\left(\mu+\sigma^{2}\right)}\right)}}{\left(\left(p_{1}(\theta+2) / \theta(\theta+1)\right)+p_{2} e^{\mu+\left(\sigma^{2} / 2\right)}\right)}$.

Some values of the mean, standard deviation, coefficient of variation, coefficient skewness, and coefficient kurtosis for the MLLND distributions are obtained for the two choices of the parameter $\theta$ and different values of the parameter $\mu$. From the results which are presented in Tables 1 and 2, we note that as $\mu$ increases, both the mean and the standard deviation increase, whereas the values of the other measures remain fairly stable.

\section{Order Statistics}

Let $X_{1}, X_{2}, \cdots, X_{m}$ be a random sample of size $m$ selected from a distribution with pdf $f(x)$ and $\operatorname{cdf} F(x)$, and also let $X_{1: m}<X_{2: m}<\cdots<X_{m: m}$ be the corresponding order statistics. The pdf of the $r$ th order statistics that say $X_{r: m}$ is given by 
$f_{r: m}(x)=\frac{m !}{(r-1) !(m-r) !}[F(x)]^{r-1}[1-F(x)]^{m-r} f(x),-\infty<x<\infty$,

and the corresponding cdf is given by

$$
\begin{aligned}
F_{(r: m)}(x) & =\sum_{j=r}^{m}\left(\begin{array}{c}
m \\
j
\end{array}\right) F^{j}(x)[1-F(x)]^{m-j} \\
& =\sum_{j=r}^{m} \sum_{k=0}^{m-j}\left(\begin{array}{c}
m \\
j
\end{array}\right)\left(\begin{array}{c}
m-j \\
k
\end{array}\right)(-1)^{k} F^{j+k}(x) .
\end{aligned}
$$

Therefore, using (25) and (26), the pdf and cdf of the $r$ th order statistics, are, respectively, given by

where

$$
\begin{gathered}
W=\left(\frac{p_{2}}{2} \operatorname{erf} c\left(\frac{\mu-\log (x)}{\sqrt{2} \sigma}\right)+p_{1}\left(1-\frac{e^{-\theta x}(\theta+\theta x+1)}{\theta+1}\right)\right)^{j-1} \times\left(-\frac{p_{2}}{2} \operatorname{erf} c\left(\frac{\mu-\log (x)}{\sqrt{2} \sigma}\right)+p_{1}\left(\frac{e^{-\theta x}(\theta+\theta x+1)}{\theta+1}-1\right)+1\right)^{m-j} \\
F_{r: m}(x, \theta, \sigma)=\frac{m ! \delta_{j-m}\left(\left(p_{2} / 2\right) \operatorname{erf} c(\mu-\log (x) / \sqrt{2} \sigma)+p_{1}\left(1-e^{-\theta x}(\theta+\theta x+1) / \theta+1\right)\right)^{j+1}}{(j+1) \Gamma(j) \Gamma(m-j+1)} .
\end{gathered}
$$

Accordingly, the density functions of the minimum and maximum order statistics, respectively, are given by

$$
\begin{aligned}
f_{1: m}(x)= & m\left(\frac{p_{1} \theta^{2}(x+1) e^{-\theta x}}{\theta+1}+\frac{p_{2} e^{-1 / 2(\log x-\mu / \sigma)^{2}}}{\sqrt{2 \pi} \sigma x}\right) \\
& \times\left(-\frac{p_{2}}{2} \operatorname{erfc}\left(\frac{\mu-\log (x)}{\sqrt{2} \sigma}\right)\right. \\
& \left.+p_{1}\left(-\left(1-\frac{e^{-\theta x}(\theta+\theta x+1)}{\theta+1}\right)\right)+1\right)^{m+1} \\
f_{m: m}(x)= & m\left(\frac{p_{1} \theta^{2}(x+1) e^{-\theta x}}{\theta+1}+\frac{p_{2} e^{-1 / 2(\log x-\mu / \sigma)^{2}}}{\sqrt{2 \pi} \sigma x}\right) \\
& \times\left(\frac{p_{2}}{2} \operatorname{erf} c\left(\frac{\mu-\log (x)}{\sqrt{2} \sigma}\right)\right. \\
& \left.+p_{1}\left(1-\frac{e^{-\theta x}(\theta+\theta x+1)}{\theta+1}\right)\right)^{m-1}
\end{aligned}
$$

\section{Maximum Likelihood Estimation}

The likelihood function (LF) for the MLLND in (1) is given by

$$
L(\Theta)=\prod_{j=1}^{n}\left[p_{1} f_{1}\left(x_{j} ; \Theta_{1}\right)+p_{2} f_{2}\left(x_{j} ; \Theta_{2}\right)\right]
$$

where $\Theta_{1}=(\theta)$ and $\Theta_{2}=(\mu, \sigma)$. By differentiating the log LF with respect to the model parameters $\Theta=\left(p_{1}, \theta, \mu, \sigma\right)$, respectively, we get the following equations

$$
\begin{aligned}
\sum_{j=1}^{n} \omega\left(x_{j} ; \Theta\right) & =0, \sum_{j=1}^{n} p_{1} \psi_{1}\left(x_{j} ; \Theta\right) \eta_{1}\left(x_{j} ; \Theta\right) \\
& =0, \sum_{j=1}^{n} p_{2} \phi_{1}\left(x_{j} ; \Theta\right) \eta_{2}\left(x_{j} ; \Theta\right) \\
& =0, \sum_{j=1}^{n} p_{2} \phi_{2}\left(x_{j} ; \Theta\right) \eta_{2}\left(x_{j} ; \Theta\right)=0,
\end{aligned}
$$

where $\omega\left(x_{j} ; \Theta\right), \psi_{1}\left(x_{j} ; \Theta\right), \eta_{1}\left(x_{j} ; \Theta\right), \eta_{2}\left(x_{j} ; \Theta\right), \phi_{1}\left(x_{j} ; \Theta\right)$, and $\phi_{2}\left(x_{j} ; \Theta\right)$ are as follows:

$$
\begin{aligned}
\omega\left(x_{j} ; \Theta\right) & =\frac{f_{1}\left(x_{j} ; \Theta_{1}\right)-f_{2}\left(x_{j} ; \Theta_{2}\right)}{f\left(x_{j} ; \Theta\right)}, \\
\psi_{1}\left(x_{j} ; \Theta\right) & =2 \theta^{-1}-x_{j}-1, \\
\eta_{1}\left(x_{j} ; \Theta\right) & =\frac{f_{1}\left(x_{j} ; \Theta_{1}\right)}{f\left(x_{j} ; \Theta\right)}, \\
\eta_{2}\left(x_{j} ; \Theta\right) & =\frac{f_{2}\left(x_{j} ; \Theta_{2}\right)}{f\left(x_{j} ; \Theta\right)}, \\
\phi_{1}\left(x_{j} ; \Theta\right) & =\frac{\left(\log x_{j}-\mu\right)}{\sigma^{2}}, \phi_{2}\left(x_{j} ; \Theta\right)=\left(\log x_{j}-\mu\right)^{2} \sigma^{-3}-\sigma^{-1},
\end{aligned}
$$


TABLE 3: MLEs for the MLLND parameters with their averages, biases, MSEs, and CIs.

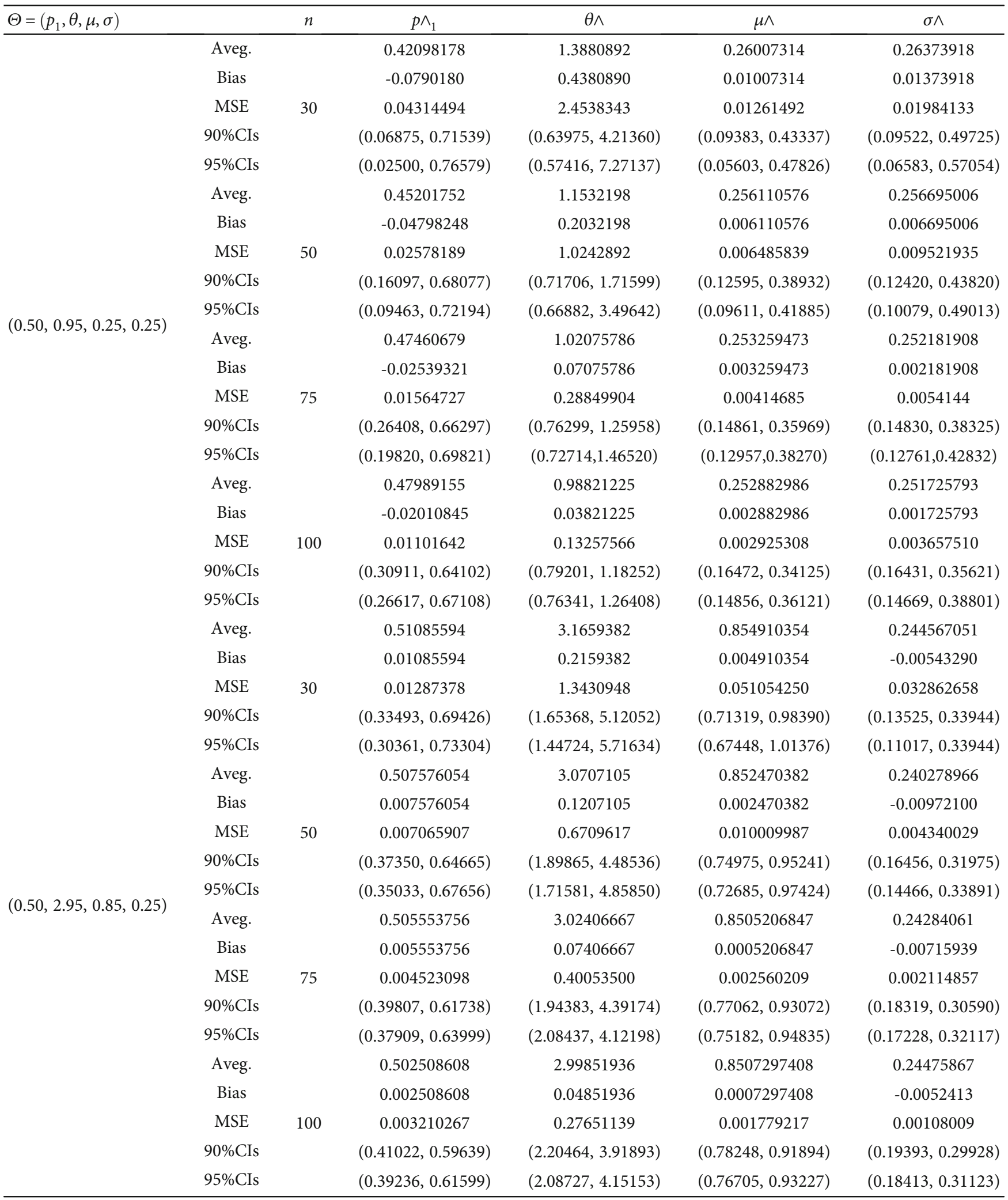


TABLE 4: Results for fitting the MLLND comparing with their components and others models.

\begin{tabular}{|c|c|c|c|c|c|c|}
\hline Models & MLEs (Std.Error) & Loglikelihood & AIC & $\mathrm{BIC}$ & KS & $p$ value \\
\hline \multirow{4}{*}{ MLLND } & $p \wedge_{1}=0.16733(0.0525)$ & -399.423 & 806.8461 & 818.2542 & 0.0344 & 0.9981 \\
\hline & $\theta \wedge=1.8819(0.7011)$ & & & & & \\
\hline & $\mu \wedge=1.9453(0.1119)$ & & & & & \\
\hline & $\sigma \wedge=0.8455(0.0852)$ & & & & & \\
\hline \multirow{3}{*}{ MTLD } & $p \wedge_{1}=0.2022(0.1150)$ & -402.4761 & 810.9522 & 819.5083 & 0.0704 & 0.5495 \\
\hline & $\theta \wedge_{1}=0.0912(0.0264)$ & & & & & \\
\hline & $\theta \wedge_{2}=0.3239(0.0483)$ & & & & & \\
\hline \multirow{4}{*}{ MLIWD } & $p \wedge_{1}=0.2374(0.0701)$ & -399.8651 & 807.7303 & 819.1384 & 0.0428 & 0.9730 \\
\hline & $\theta \wedge=1.2975(0.4920)$ & & & & & \\
\hline & $\alpha \wedge=0.1804(0.0221)$ & & & & & \\
\hline & $\beta \wedge=1.5473(0.1528)$ & & & & & \\
\hline \multirow{5}{*}{ MTWD } & $p \wedge_{1}=0.9554(0.0438)$ & -401.2711 & 812.5422 & 826.8023 & 0.0584 & 0.7746 \\
\hline & $\alpha \wedge_{1}=8.8542(1.0315)$ & & & & & \\
\hline & $\alpha \wedge_{2}=0.4747(0.2526)$ & & & & & \\
\hline & $\beta \wedge_{1}=0.9751(0.0811)$ & & & & & \\
\hline & $\beta \wedge_{2}=2.5650(1.9307)$ & & & & & \\
\hline \multirow{5}{*}{ MIWWD } & $p \wedge_{1}=0.7344(0.0680)$ & -398.7583 & 807.5166 & 821.7768 & 0.0439 & 0.9655 \\
\hline & $\alpha \wedge_{1}=0.1879(0.0166)$ & & & & & \\
\hline & $\alpha \wedge_{2}=1.7452(0.5879)$ & & & & & \\
\hline & $\beta \wedge_{1}=1.5208(0.1446)$ & & & & & \\
\hline & $\beta \wedge_{2}=0.8896(0.1764)$ & & & & & \\
\hline \multirow{2}{*}{ LND } & $\mu=1.5111 \wedge(0.1133)$ & -406.8025 & 817.6050 & 823.3091 & 0.0998 & 0.1556 \\
\hline & $\sigma=1.2818 \wedge(0.0801)$ & & & & & \\
\hline LD & $\theta=0.2129 \wedge(0.0134)$ & -417.9239 & 837.8477 & 840.6997 & 0.1335 & 0.0207 \\
\hline
\end{tabular}

and $f\left(x_{j} ; \Theta\right), f_{1}\left(x_{j} ; \Theta_{1}\right)$, and $f_{2}\left(x_{j} ; \Theta_{2}\right)$ are as in $(1,2,3)$, respectively. The MLEs of the parameters can be obtained by solving systems of nonlinear Eqs. given in (33) using the package nleqslv() in $R$.

The numerical results are obtained in Table 3 for two different combinations of the parameters. The first one corresponds to a unimodal distribution, whereas the second choice is for bimodal distribution.

In each case, the averages of the MLEs, biases, mean squared errors (MSE), and the lower and upper limits of the $95 \%$ nd $90 \%$ confidence intervals (CIs) for the parameters are computed at different sample sizes.

It is clear from Table 3 that the MSE decreases as the sample size increases for all estimates parameters. Furthermore, the values of the bias decrease. Also, as the sample size increases, the width of the confidence intervals (CIs) for the parameters decreases. The number of replications of the simulation results is taken to 10000 .

\section{Application}

The flexibility of the proposed model is illustrated by applying it on a real data set given in Shanker et al. [25]. It represents the remission times (in months) of sample size $n=128$ bladder cancer patients as reported in Lee and Wang [26].
This data was previously analyzed by Daghestani et al. [18], who compared their proposed model, mixture of Lindley and Weibull distribution MLWD to two other models; mixture of two one- parameter Lindley distribution MTLD and mixture of two Weibull distribution MTWD. They showed that their model provides the best fit as it has the lowest values of the KS statistic and AIC criterion and the highest $p$ value.

In this paper, we compared our proposed model with six other models including the three models given in Daghestani et al. [18] and there other models, namely, the mixture of Lindley inverse Weibull distributions (MLIWD), mixture of inverse Weibull and Weibull distributions (MIWWD), one component Lindley distribution, and one component lognormal distribution. All the seven models are fitted to the real data. The results are listed in Table 4. Table 4 shows the MLEs of the parameters of the seven models with their standard errors and values of the KS statistic which is used to assess the similarity between the actual data and the fitted distributions. In the $R$ software, the packages (MASS) and (fitdistrplus) are used to calculate the values of KS statistics and their corresponding $R$ values for the seven distributions. The loglikelihood function and some criteria that measure the quality of the fitted models such as AIC and BIC criteria are computed. Table 5 gives the results of the variance 


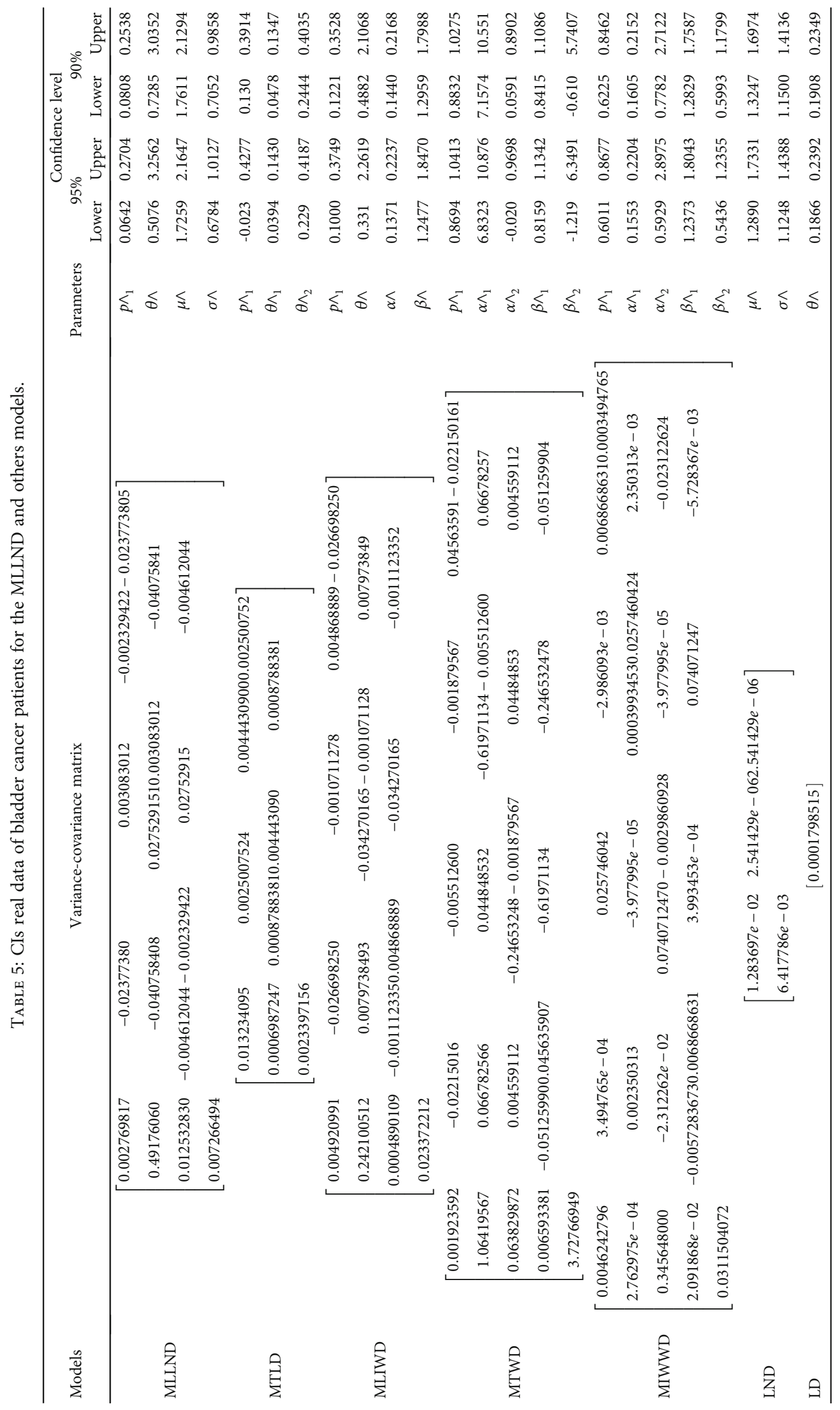


Histogram and theoretical densities

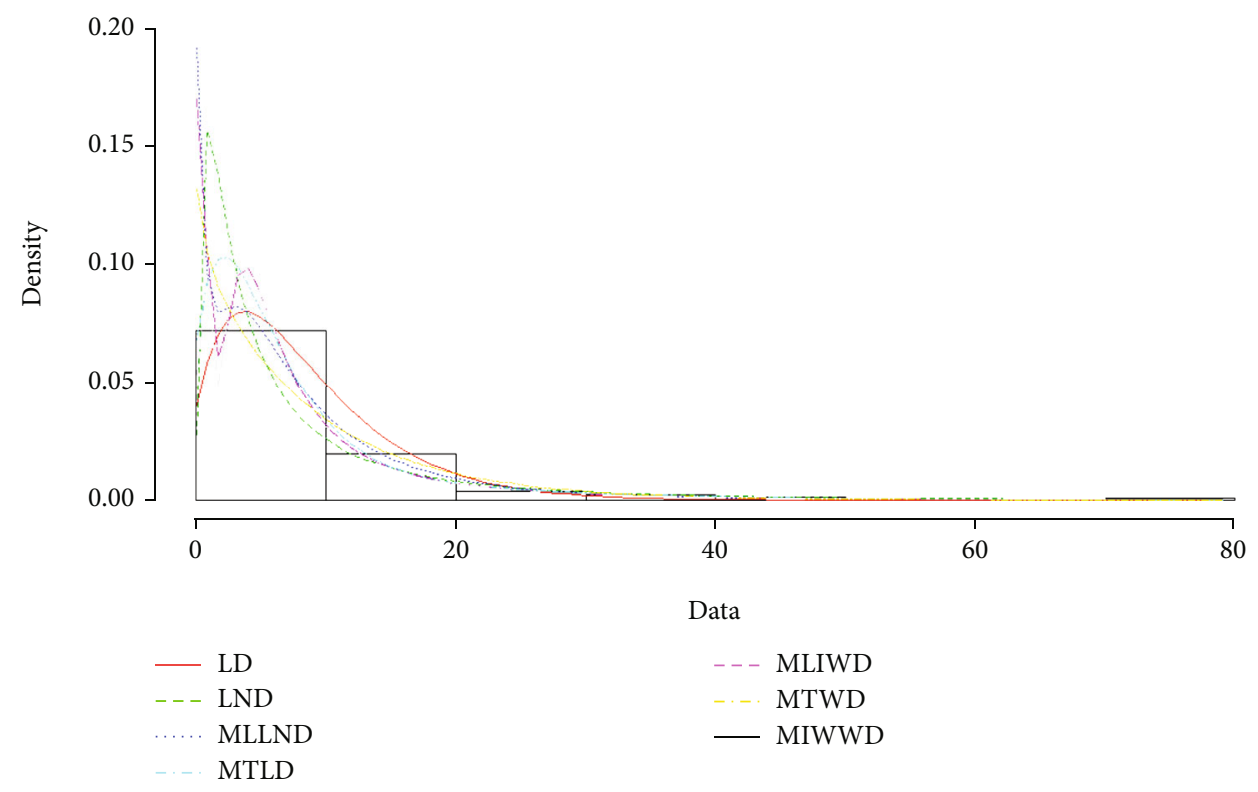

FIgURE 2: Comparison of different pdfs of the data application.

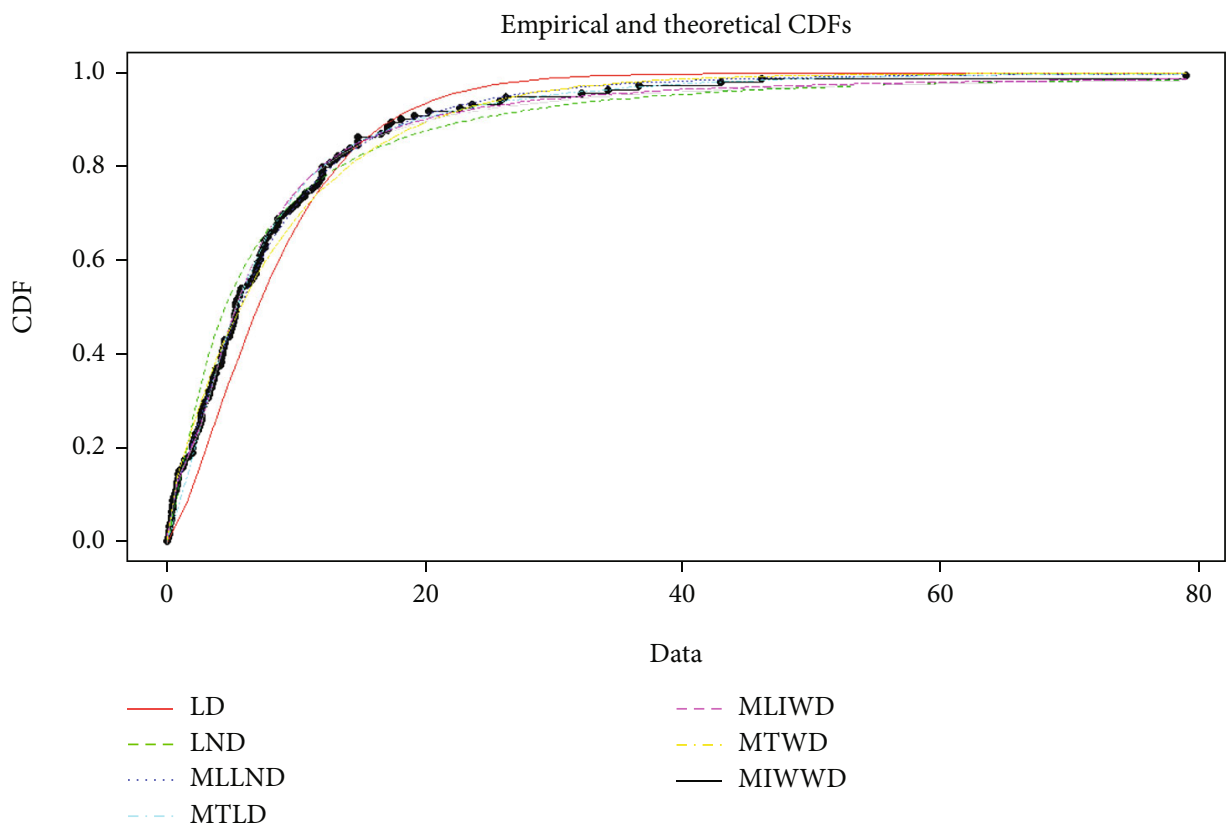

Figure 3: Comparison of different CDFs of the data application.

covariance matrices for the competitive models calculated by the function $\operatorname{vcov}()$ in $R$ which depends on the package fit$\operatorname{dist}()$. Lower and upper limits of the $95 \%$ nd $90 \%$ confidence intervals (CIs) for the parameters of the different distributions are also provided. Figure 2 displays the plots of the pdfs of the seven fitted models superimposed on the histogram of the real data set by using the function $\operatorname{denscomp}()$ in $R$. The figure shows that the MLLND provides a very good fit for these data compared to other mixtures and one component models. Figure 3 shows the comparisons of the plots of the theoretical cdfs of the fitted distributions to the empirical cdf of the data using the function $\operatorname{cdfcomp}()$ in $R$. Again, it is clear that the cdf of the MLLND is closer to the empirical distribution than any other model. Figures 4 and 5 show the pp plots and qq plots for the real data to those of the compared models using the functions ppcomp () and qqcomp(), respectively, in $R$. The plots show the adequency of the proposed model to fit the real data compared to other models. 


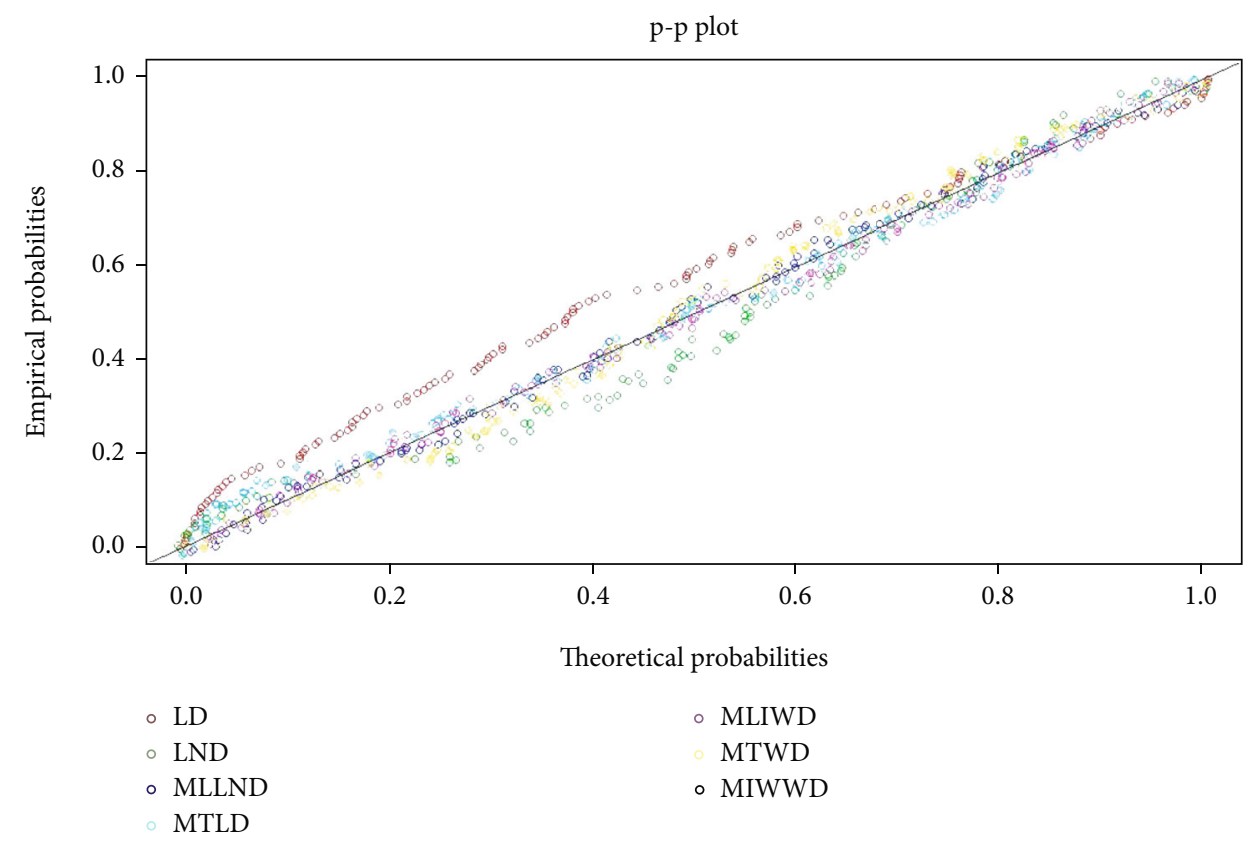

FIgURE 4: Comparison of different pp plots of the data application.

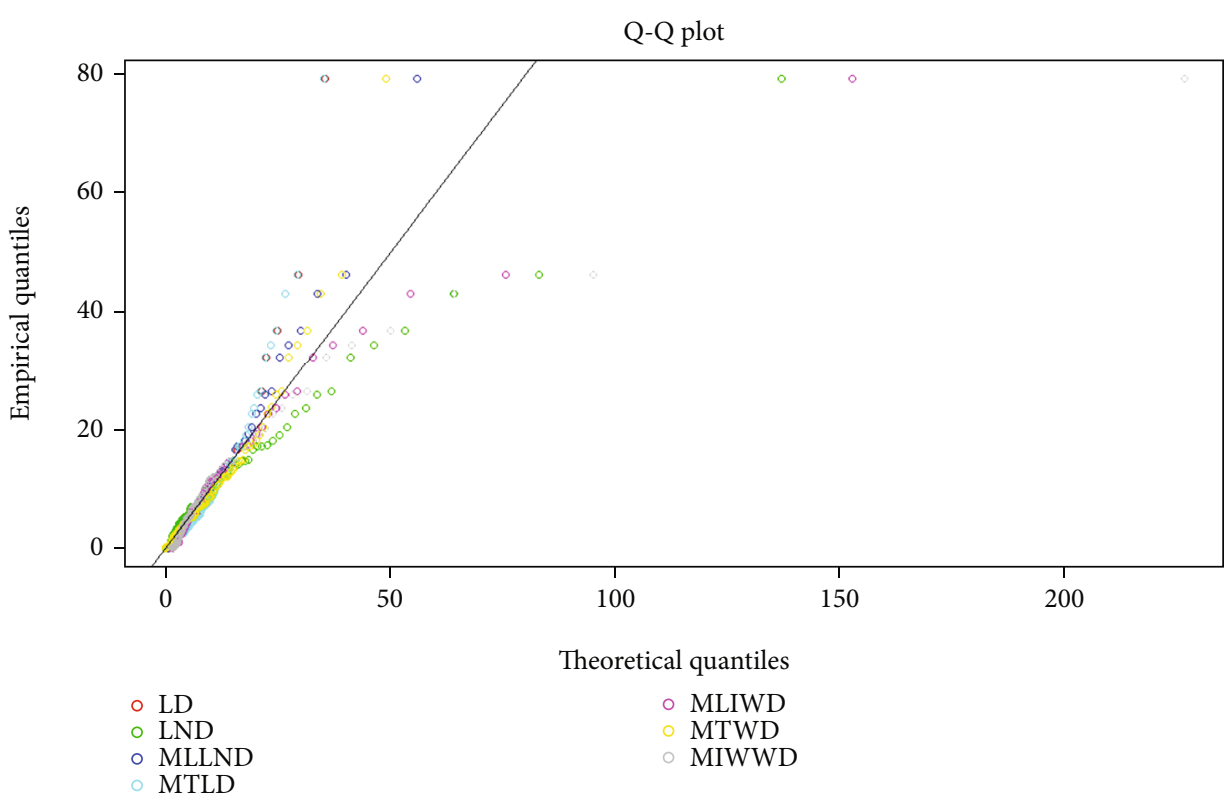

FIgURE 5: Comparison of different qq plots of the data application.

In short, all the above figures indicate that the MLLND is the perfect fit for the real data set compared to all the competitive models.

\section{Concluding Remarks}

This paper introduces a new mixture model which is the MLLND to handle heterogeneous data. This model was proposed due to the importance of each of the Lindley and lognormal distributions and their great applications, and so it was expected that mixing these two distributions together would lead to a more flexible model than its com- ponents distributions. Some properties of the MLLND were obtained such as the expectation, the mean, variance, the mode (s), median, reliability function, HRF, skewness, kurtosis, and coefficient of variation. The pdf for the minimum and maximum order statistics of the MLLND is presented. Maximum likelihood estimation of the parameters of the model was discussed and estimated via simulation with number of replications 10000 runs. The main objective of this paper was to illustrate the applicability of the proposed distribution compared to six competitive distributions. This was achieved by showing the ability of the model to fit a well-known real data set better than the compared models. 
This was done by using the formal test like $K$ - $S$ statistic as well as information criteria and also through graphical procedures such as plots of theoretical and empirical cdfs, pp plots, and qq plots.

\section{Abbreviations}

MLLND: Mixture of Lindley and lognormal distributions MTLD: $\quad$ Mixture of two Lindley distributions

MLIWD: Mixture of Lindley inverse Weibull distributions; mixture of two Weibull distributions (MTWD)

MIWWD: Mixture of inverse Weibull Weibull distributions

LD: $\quad$ One component of Lindley distribution

LND: One component from lognormal distribution

MLEs: Maximum likelihood estimates

LF: $\quad$ Likelihood function

pdf: $\quad$ Probability density function

cdf: $\quad$ Cumulative distribution function

HRF: Hazard rate function

Sk: $\quad$ Coefficient of skewness

Ku: $\quad$ Coefficient of kurtosis

Cv: $\quad$ Coefficient of variation

CIs: $\quad$ Confidence intervals

AIC: $\quad$ Akaike information criterion

KS: Kolmogorov-Smirnov

ECDF: Empirical cumulative distribution function

pp: $\quad$ Probability plot

qq plot: Quantile quantile plot

Std.: $\quad$ Error: standard error

BIC: $\quad$ Bayesian information criterion.

\section{Data Availability}

The data used to support the findings of this study have been deposited in the Shanker et al. [26] repository ([doi:10 $.15406 /$ bbij.2016.03.00061]).

\section{Conflicts of Interest}

The author declares that there is no conflict of interests regarding the publication of this paper.

\section{References}

[1] B. S. Everitt and D. J. Hand, Finite Mixture Distributions, Chapman \& Hall, London, England, 1981.

[2] G. McLachlan and D. Peel, Finite Mixture Models, John Wiley \& Sons, New York, 2000.

[3] G. J. McLachlan and K. E. Basford, Mixture Models: Applications to Clustering, Marcel Dekker, New York, 1988.

[4] D. M. Titterington, A. F. M. Smith, and U. E. Makov, Statistical Analysis of Finite Mixture Distributions, John Wiley \& Sons, Chichester, England. w Jersey, 1985.

[5] B. G. Lindsay, Mixture Models: Theory, Geometry and Applications, The Institute of Mathematical Statistics, Hayward, CA, 1995.

[6] G. J. McLachlan and T. Krishnan, The EM Algorithm and Extensions, John Wiley \& Sons, New York, 1997.

[7] A. S. Al-Moisheer, K. S. Sultan, and M. A. Al-Shehri, "A Mixture of Inverse Weibull and Inverse Burr Distributions:
Properties, Estimation, and Fitting," Mathematical Problems in Engineering, vol. 2017, Article ID 7824323, 11 pages, 2017.

[8] A. S. Al-Moisheer, R. M. Alotaibi, G. A. Alomani, and H. Rezk, "Bivariate mixture of inverse Weibull distribution: properties and estimation," Mathematical Problems in Engineering, vol. 2020, Article ID 5234601, 12 pages, 2020.

[9] A. S. Al-Moisheer, "Homogeneity tests for Burr III mixture model," Journal of Computational and Theoretical Nanoscience, vol. 15, no. 8, pp. 2515-2520, 2018.

[10] A. S. Al-Moisheer, "Sequential test for a mixture of finite exponential distribution," Journal of Mathematics, vol. 2021, Article ID 6625853, 10 pages, 2021.

[11] D. V. Lindley, "Fiducial distributions and Bayes' theorem," Journal of the Royal Statistical Society: Series B, vol. 20, no. 1, pp. 102-107, 1958.

[12] D. V. Lindley, “Approximate Bayesian methods," Trabajos de Estadistica $Y$ de Investigacion Operativa, vol. 31, no. 1, pp. 223-245, 1980.

[13] M. E. Ghitany, B. Atieh, and S. Nadarajah, "Lindley distribution and its application," Mathematics and Computers in Simulation, vol. 78, no. 4, pp. 493-506, 2008.

[14] J. S. Kim and B. J. Yum, "Selection between Weibull and lognormal distributions: a comparative simulation study," Computational Statistics \& Data Analysis, vol. 53, no. 2, pp. 477-485, 2008.

[15] C. T. Lin, S. J. S. Wu, and N. Balakrishnan, "Planning life tests with progressively Type-I interval censored data from the lognormal distribution," Journal of Statistical Planning and Inference, vol. 139, no. 1, pp. 54-61, 2009.

[16] A. S. Al-Moisheer, A. F. Daghestani, and K. S. Sultan, "Mixture of two one-parameter Lindley distributions: properties and estimation," Journal of Statistical Theory And Practice, vol. 15, no. 1, 2021.

[17] A. S. Al-Moisheer, A. F. Daghestani, and K. S. Sultan, "Mixture of lindley and inverse Weibull distributions: properties and estimation," WSEAS Transactions on Mathematics, vol. 20, pp. 134-143, 2021.

[18] A. F. Daghestani, K. S. Sultan, and A. S. Al-Moisheer, "Mixture of lindley and Weibull distributions: properties and estimation," Journal of Statistics Applications \& Probability, vol. 10, no. 2, pp. 301-313, 2021.

[19] R. Shanker, S. Sharma, and R. Shanker, "A two-parameter Lindley distribution for modeling waiting and survival times data," Applied Mathematics, vol. 4, no. 2, pp. 363-368, 2013.

[20] E. L. Crow and K. Shimizu, Eds., The Lognormal Distribution, Marcel Dekker, New York, 1988.

[21] N. L. Johnson, S. Kotz, and N. Balakrishnan, Continuous Univariate Distributions, Vol. 1, John Wiley \& Sons, New York, 2nd edition, 1994.

[22] E. K. Al-Hussaini and K. S. Sultan, "Reliability and hazard based on finite mixture models," in Handbook of StatisticsVol. 20, N. Balakrishnan and C. R. Rao, Eds., pp. 139-183, Elsevier, Amsterdam, 2001.

[23] K. S. Sultan, Mixtures of lognormal distributions, [Ph.D. thesis], University of Assiut, Egypt, 1991.

[24] K. S. Sultan and A. S. Al-Moisheer, "Mixture of Inverse Weibull and Lognormal Distributions: Properties, Estimation, and Illustration," Mathematical problems in Engineering, vol. 2015, Article ID 526786, 8 pages, 2015. 
[25] R. Shanker, H. Fesshaye, and S. Selvaraj, "On modeling of lifetime data using one parameter Akash, Lindley and exponential distributions," Biometrics \& Biostatistics International Journal, vol. 3, no. 2, 2016.

[26] E. T. Lee and J. W. Wang, Statistical Methods for Survival Data Analysis, John Wiley \& Sons, Incorporated, Hoboken, New Jersey, 2003. 